\title{
Engineering of Microstructures
}

\author{
R.T. DeHoff \\ Department of Materials Science and Engineering, \\ University of Florida, Gainesville, Florida
}

Received: August 15, 1998; Revised: March 30, 1999

\begin{abstract}
Structure is at the heart of the materials science paradigm connecting processing with properties. In the hierarchy of structures that exist in materials microstructure offers the richest variety of structural arrangements. This variety is often conveniently accessible, e.g., simply by heat treatment or mechanical deformation. Exploration of the relation between properties and microstructure serves to establish a target range of microstructural states that will perform. In order to attain a target microstructure it is necessary to understand what microstructures are, and how they evolve in processing. This presentation focuses upon the set of tools that must be combined to achieve this control:

1. Geometry

2 Thermodynamics

3. Kinematics

4. Kinetics.

The content of these tools is reviewed briefly and their uses illustrated in developing an understanding of how microstructures evolve. In this development an attempt is made to carry the description of each microstructural process as far as possible without making simplifying assumptions. The study of microstructures with this rigorous point of view was termed by F.N. Rhines, "microstructology".
\end{abstract}

\section{Keywords: microstructure}

\section{Introduction}

Geometry. The spatial distribution of things. The heart of the materials science paradigm, Fig. 1. Processing determines structure; structure determines properties. Structure is the spatial distribution of things seen and unseen.

The scientific content of materials science is based upon a hierarchy of structures:

Nuclear structure determines nuclear properties.

Atomic structure consists of a spatial distribution of subatomic particles.

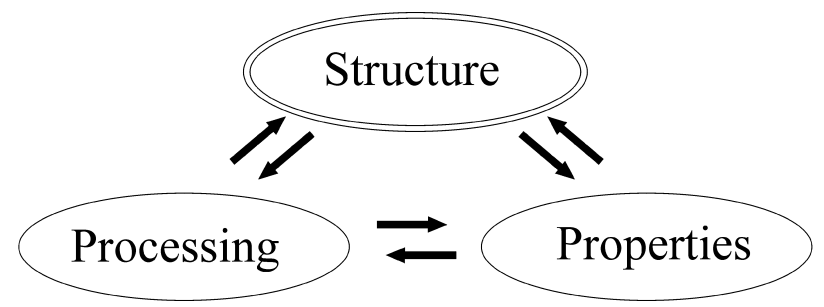

Figure 1. The matrials science paradigm shows that structure connects processing with properties.
Molecular structure extends this spatial distribution to groups of atoms and dominates the chemical behavior of the molecules.

Crystal structure for metals, alloys, ceramics and polymers is the spatial distribution of atoms on three dimensional lattices.

Electronic structure is visualized as the spatial distribution of the probability density of an electron cloud, is conceived for atoms, molecules and crystals.

Defect structure in crystals includes point, line and surface defects.

Microstructure influences virtually all aspects of the behavior of materials.

Macrostructure is the spatial distribution of material in the useful object that is the goal of the endeavor.

Materials science first adapted from physics or devised the conceptual content of these structures, then discovered ways to obtain information about that content, and is now learning to use that information to predict the response of materials to processing, and the properties that the processed material exhibits. 
For a given piece of material microstructure is the most richly variable, and thus the most susceptible to control, of the elements in the materials structural hierarchy. Further, access to this richness is frequently conveniently available through very simple processes. For example a complete revision of the microstructure of a material may be achieved by simply changing its temperature. It is this rich variability, the attendant potential to produce a particular microstructural state with desired properties, and the ease with which this control can frequently be achieved, that puts microstructure at the focal point of materials science.

This paper deals with half of the materials science paradigm: the selection of the material and processing combination with the objective of engineering a target microstructural state. The other half of the paradigm, the connection of microstructure to properties and performance so that the nature of the target microstructural state may be identified, is a subject of equal importance, sophistication and complexity. However, this important subject is outside the scope of this presentation.

A breadth of experience with particular materials and the accumulating information and understanding about the operation of the components in the hierarchy of structures has brought the field to the point where microstructures can be produced by design. Once the realm of the target microstructure has been identified, the key to the design of microstructures is an understanding of microstructural evolution. This requires an understanding of the processes that are involved in bringing about a change in the spatial distribution of the geometric elements that make up a microstructure. This paper explores the tools that are required to understand how microstructures evolve. Rhines has termed the development of these tools and their knowledgeable application to understand how microstructures arrive at a given state microstructology ${ }^{1}$. He believed that those who study microstructures should strive to be quantitative in this endeavor and to take the application of these tools as far as is possible before introducing simplifying assumptions. These tools are:

1. Geometric concepts that describe the microstructural state, quantified by the application of stereology;

2. Thermodynamics and its implementation in phase diagrams which provide the context within which processes occur;

3. Kinematics of evolving microstructures that relate local boundary motions to global geometric change; and

4. Kinetics of the processes that operate in the kinematics.

The role of each of these tools in the design process will be examined. Of course the tool that finds the most use in microstructural design is the microscope, whether it be TEM, SEM, the light microscope, or other versions of instruments that produce images of microstructures.
The first decision that will be made in the design process is a specification of the chemistry of the material. This primal decision has the widest range of variables of all of the choices that are made in microstructural design. How many elements, which elements, and how much of each has a profound effect on all subsequent possibilities. All of the components in the materials structural hierarchy are circumscribed first by this list of elements, and their relative amounts, from which the material is made. Ultimately, these elements are gleaned from the earth's crust. The very sophisticated and heroic processes that convert ores into materials are beyond the scope of this presentation. Our interest is focused upon structural evolution in solidification and in the solid state that follows.

The "microstructure" begins with the first nuclei of the solid phase that forms in the liquid melt. The subsequent path of microstructural evolution for the material is highly variable depending on the sequence of thermal, mechanical, chemical and physical environments in which the material is placed. The microstructural state at each point along the path is the precursor to each subsequent state. And so the system evolves.

This development begins with a discussion of the concept of the microstructural state of a given material. A given microstructural state is a point that lies along a path of microstructural evolution. Possible paths evolving from a state are circumscribed by the thermodynamics of the alloy system reflected mostly in its phase diagram. The specific microstructural path from some initial state is then determined by the processing applied to the system. The geometric change that describes this path is described by a combination of topological events ${ }^{1}$ and the motion of interfaces that delineate the constituents in the microstructure. The kinematics of microstructural change relates rates of change of the geometric properties in the microstructural state to the distribution of interface velocities. Finally, the rates of topological processes and the distribution of interface velocities are determined by the kinetics of the controlling processes. These four fields of scientific endeavor, - geometry, thermodynamics, kinematics and kinetics, must be combined in order to achieve a sophisticated level of understanding of the connection of processing to structure in materials science.

\section{Geometry and the Microstructural State}

Microstructures are three-dimensional space-filling, not-regular, not-random distributions of phases and their boundaries. The geometric state of a microstructure may be reported at three levels ${ }^{2}$ :

1. The qualitative microstructural state;

2. The quantitative microstructural state; and

3. The topographic microstructural state. 


\subsection{The qualitative microstructural state}

A microstructure is a three dimensional entity; features in the microstructure may have 3,2 , 1 , or zero dimensions. That is, features may be volumes (grains, particles), surfaces (grain boundaries, interfaces), lines (triple lines, edges) or points (quadruple points, vertices on polyhedral particles). The qualitative microstructural state for a microstructure is comprised of a list of all of the phases that it contains (with their compositions and crystal structures) together with a list of all of the classes of 3,2,1 and 0 dimensional features that are found in the structure. For example, Fig. 2 sketches a microstructure with particles of a $\beta$ phase distributed in the grain boundary network of the $\alpha$ grains. This structure has $\alpha$ and $\beta$-type volumes (the three dimensional phases); $\alpha \alpha$ and $\alpha \beta$ interfaces, $\alpha \alpha \alpha, \alpha \alpha \beta$ triple lines and $\alpha \alpha \alpha \alpha, \alpha \alpha \alpha \beta$ quadruple points ${ }^{2}$. The nickel based superalloy structure sketched in Fig. 3 has $\gamma$ and $\gamma$ ' phase in the volumes of the grains and oxides, carbides and borides along the grain boundary. A complete feature list for this system containing all of the classes of interfaces and triple lines has over 30 entries.

The specification of the qualitative microstructural state may be further embellished with descriptive words that call to mind familiar shapes like platelet, acicular, spheroidal, dendritic, lamellar, etc., or suggest other aspects of the structure like sparse or space filling.

\subsection{The quantitative microstructural state}

Each of the features in the qualitative microstructural state has associated with it one or more geometric properties. These properties may be defined for individual features in a collection. They also have global counterparts that describe a complete collection of features. For example, in a collection of particles of a $\beta$ phase distributed in a structure, each particle has a value of its volume. The complete set of $\beta$ particles also has a value for its volume. Specification of the quantitative microstructural state is initiated when one of its geometric properties is measured.

Volume is a familiar example of a geometric property that has unambiguous meaning for real three dimensional

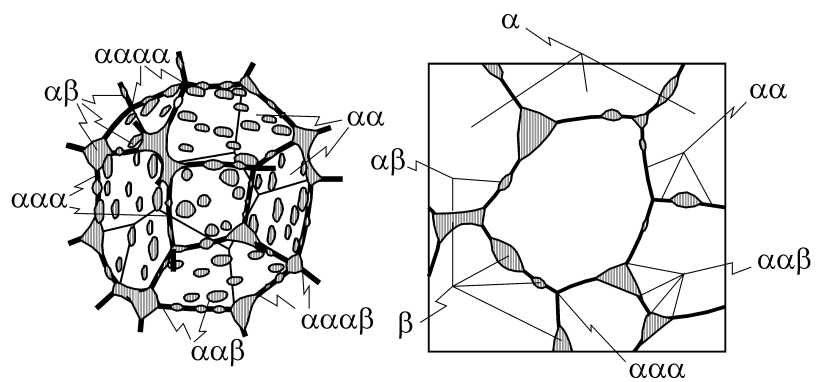

Figure 2. Microstructure with $\beta$ phase particles distributed on the grain boundary network in the $\alpha$ phase, displaying $\alpha \alpha$ and $\alpha \beta$ interfaces, and $\alpha \alpha \alpha$ and $\alpha \alpha \beta$ triple lines. features regardless of their shape, size or size distribution. There exists a collection of geometric properties that may be thought of as fundamental because they have this attribute. Indeed, it is no accident that the geometric properties that are accessible to measurement through the methods of stereology are precisely those that have general and unambiguous meaning for real three dimensional microstructures. These fundamental properties fall into the following categories:

1. Topological properties;

2. Metric properties;

a. Measures of extent;

b. Curvature measures.

Properties are usually reported in a normalized format. For example, the volume of particles of a set of features is usually reported as the volume of those features per unit volume of structure. Thus, the measure of extent of three dimensional features is reported as the volume fraction, written $\mathrm{V}_{\mathrm{V}}$, occupied by the feature set. These normalized geometric properties are listed in Table 1, along with the notation used to describe each property value.

If a property of a feature (be it $0,1,2$ or 3-dimensional) is unchanged if the feature is stretched or distorted in a completely arbitrary way, so long as it is not severed or joined to itself in the deformation, then that property is a topological property. The most familiar topological property is the number, $\mathrm{N}$, of disconnected parts of a feature. It is easy to visualize the number of (three dimensional) grains in a polycrystal (Not so easy to measure it!), or the number of (two dimensional) grain faces, or the number of grain edge segments. Connectivity, $\mathrm{C}$, reports the number of cuts that can be made through the feature without separating it into two parts ${ }^{3}$. The pore phase in a lightly sintered

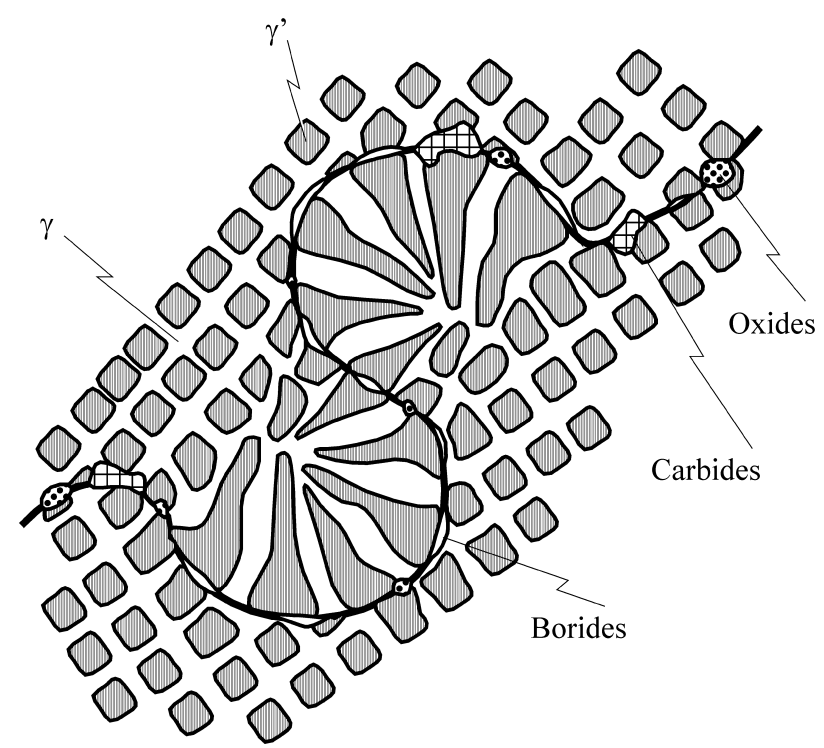

Figure 3. A microstructure for a $\gamma / \gamma^{\prime}$ superalloy with oxides, borides and carbides at the grain boundaries has a very long feature list. 
Table 1. Feature list table: three dimensional structures.

\begin{tabular}{|c|c|c|c|c|}
\hline Dim. & Description & One phase & Two phases & Three phases \\
\hline 3 & Volume & $\alpha$ & $\alpha, \beta$ & $\alpha, \beta, \gamma$ \\
\hline 2 & Surface & $\alpha \alpha$ & $\alpha \alpha, \alpha \beta, \beta \beta$ & $\alpha \alpha, \alpha \beta, \alpha \gamma, \beta \beta, \beta \gamma, \gamma \gamma$ \\
\hline 1 & Lines & $\alpha \alpha \alpha$ & $\begin{array}{c}\alpha \alpha \alpha, \alpha \alpha \beta, \alpha \beta \beta \\
\beta \beta \beta\end{array}$ & $\begin{array}{c}\alpha \alpha \alpha, \alpha \alpha \beta, \alpha \alpha \gamma, \alpha \beta \beta, \\
\alpha \beta \gamma, \beta \beta \beta, \beta \beta \gamma, \beta \gamma \gamma \\
\gamma \gamma\end{array}$ \\
\hline 0 & Points & $\alpha \alpha \alpha \alpha$ & $\begin{array}{c}\alpha \alpha \alpha \alpha, \alpha \alpha \alpha \beta \\
\alpha \alpha \beta \beta, \alpha \beta \beta \beta \\
\beta \beta \beta \beta\end{array}$ & $\begin{array}{c}\alpha \alpha \alpha \alpha, \alpha \alpha \alpha \beta, \alpha \alpha \alpha \gamma, \\
\alpha \alpha \beta \beta, \alpha \alpha \beta \gamma, \alpha \alpha \gamma \gamma, \\
\alpha \beta \beta \beta, \alpha \beta \beta \gamma, \alpha \beta \gamma \gamma, \\
\alpha \gamma \gamma \gamma, \beta \beta \beta \beta, \beta \beta \beta \gamma \\
\beta \beta \gamma \gamma, \beta \gamma \gamma, \gamma \gamma \gamma \gamma\end{array}$ \\
\hline
\end{tabular}

powder stack has a very high value of connectivity ${ }^{4}$, depending on the particle size and the complexity of the powder particles. Its connectivity decreases with densification. The grain edge network in a polycrystal has a high connectivity. Topological properties provide rudimentary information about the skeleton of the phases in a microstructure.

Metric properties have values that depend explicitly upon the shape and size of the features being described. There are two subcategories: measures of extent and curvature measures.

The metric measures of extent are the most familiar of all fundamental geometric properties. They report a quantitative measure of how much of the feature exists in the structure. Volume, V, reports this for three dimensional features, surface area, $\mathrm{S}$, reports it for two dimensional features and length, L, reports it for one dimensional features. These are the geometric properties of microstructures that are most often quantified. Because these concepts are familiar, measured results are most easily interpreted.

Metric curvature measures have a more sophisticated meaning, but are indeed fundamental in the sense that they have values that provide unambiguous meaning for features of arbitrary geometry. Curvature is a local property that is defined at a point on a surface, or on a space curve. The curvature measures that are accessible are global properties obtained by summing (integrating) the local values of curvature over the feature ${ }^{5}$. Total curvatures may thus be defined for surfaces ${ }^{5-9}$ (by integrating the local mean curvatures over the area of the surface to produce the integral mean curvature, $\mathrm{M}$ ) and lines ${ }^{10}$ (by integrating the local line curvature along the line, K). The sophisticated content of these concepts makes difficult the visualization of the meaning of the resulting numbers except in limiting cases. For example, the integral mean curvature, $\mathrm{M}$, of a collection of platelets or flakes reports the total length of the edges of those objects ${ }^{8,9}$. The fact that these global properties are fundamental in the sense described in this section, and that they may be measured using simple stereological methods makes their study important.

Averages of these properties may be defined by combining them as shown in Table 2. For example, take any global measure of extent and divide by the number of features to produce the number weighted average of that property. The average volume of particles in an array is just $\mathrm{V} / \mathrm{N}$; the average surface area is $\mathrm{S} / \mathrm{N}$. Other average measures may be defined.

The mean lineal intercept of features $\langle\lambda\rangle^{11,12}$ (the average surface-to-surface distance through features in the structure, Fig. 4, is widely used to characterize the scale of those features in a microstructure. This property is fundamental and is given by

$$
<\lambda>=\frac{4 V}{S}
$$

This measure of scale must be used knowledgeably. For example, for platelets $\langle\lambda\rangle$ is twice their average thickness, but provides no information about the scale of the structure in the plane of the plates ${ }^{8}$. For rods, $<\lambda>$ is twice the average diameter, but contains no information about the average rod

Table 2. Geometric properties of feature sets.

\begin{tabular}{|c|c|c|}
\hline Features & Geometric Property & Symbol/Units \\
\hline & $\begin{array}{l}\text { Topological } \\
\text { Properties }\end{array}$ & \\
\hline$\alpha, \beta \ldots$ & Number density & $\mathrm{N}_{\mathrm{V}}\left(\mathrm{m}^{-3}\right)$ \\
\hline \multirow[t]{2}{*}{$\alpha, \beta \ldots$} & $\begin{array}{l}\text { Connectivity } \\
\text { density }\end{array}$ & $\mathrm{C}_{\mathrm{V}}\left(\mathrm{m}^{-3}\right)$ \\
\hline & Metric properties & \\
\hline$\alpha, \beta \ldots$ & Volume fraction & $V_{V}\left(m^{3} / m^{3}\right)$ \\
\hline$\alpha \alpha, \alpha \beta \ldots$ & Surface area density & $\mathrm{S}_{\mathrm{V}}\left(\mathrm{m}^{2} / \mathrm{m}^{3}\right)$ \\
\hline$\alpha \alpha, \alpha \beta \ldots$ & $\begin{array}{l}\text { Integral mean } \\
\text { curvature }\end{array}$ & $M_{V}\left(m / m^{3}\right)$ \\
\hline$\alpha \alpha \alpha, \alpha \alpha p \ldots$ & Length density & $\operatorname{Lv}\left(\mathrm{m} / \mathrm{m}^{3}\right)$ \\
\hline
\end{tabular}




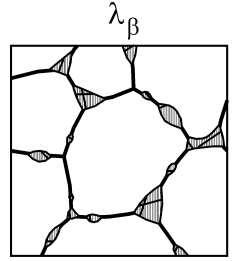

(a)

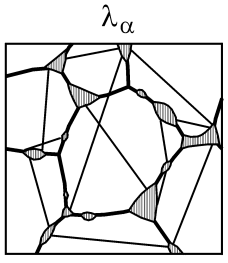

(b)

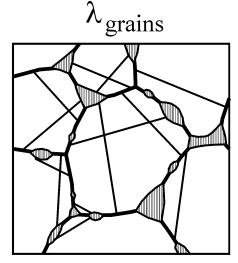

(c)
Figure 4. Illustration of the meaning of various measures of $\langle\lambda\rangle$, the mean lineal intercept in a two phase microstructure structure: (a) $\lambda_{\beta}$ is the mean lineal intercept of the $\beta$ phase; (b) $\lambda_{\alpha}$ is the mean intercept of the $\alpha$ phase (the mean free path between $\beta$ particles); (c) $\lambda_{\text {grains }}$ is the mean intercept of a grains.

length ${ }^{8}$. The mean lineal intercept is the most popular measure of grain size in polycrystals.

The average mean surface curvature ${ }^{7},<\mathrm{H}>$, is given by

$$
<\mathrm{H}>=\frac{\mathrm{M}}{\mathrm{S}}
$$

This property becomes important in structures in which capillarity effects play a key role, since the local mean curvature is the geometric property at the focus of the thermodynamic behavior of curved surfaces ${ }^{13}$. The dihedral angle at edges in a structure also plays a key role in capillarity driven behavior in microstructures. The average dihedral angle, $<\chi>$ is a fundamental property that may be defined and measured ${ }^{10}$.

The distribution of geometric property values within a feature set may be visualized in terms of fundamental variables, since these properties can be defined for each

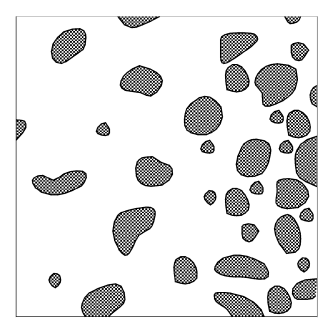

(a)

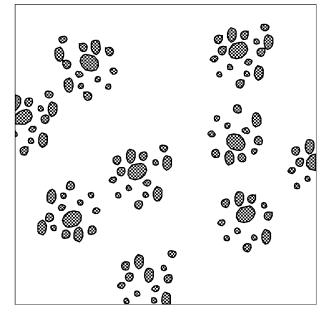

(c)

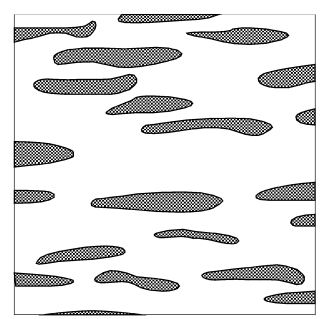

(b)

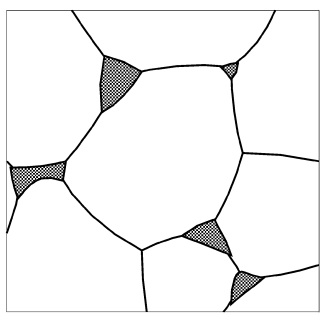

(d)
Figure 5. Microstructures illustrating the concepts of (a) gradients, (b) anisotropies, and (c) associations.
Table 2.Features you see on a section through the microstructure.

\begin{tabular}{lcc}
\hline Feature in 3-D & Designation & Feature in 2-D \\
\hline $\begin{array}{l}\text { Volume } \\
\text { Grain }\end{array}$ & $\alpha$ & Area \\
Particle & $\beta$ & Grain (section) \\
Particle (section) & Line \\
Surface & & Grain boundary (trace) \\
Interface & $\alpha \alpha, \beta \beta$ & Phase boundary (trace) \\
Line (space curve) & $\alpha \beta$ & Point \\
Grain edge (triple & $\alpha \alpha \alpha$ & Triple point \\
line) & $\alpha \alpha \beta, \alpha \beta \beta$ & Triple point \\
Triple lines & & \\
Point & & \\
Quadruple point & $\alpha \alpha \alpha \alpha$ & Not observed \\
Quadruple points & $\alpha \alpha \alpha \beta$ & " " " \\
& $\alpha \alpha \beta \beta$ & " " " \\
& $\alpha \beta \beta \beta$ & \\
\end{tabular}

feature in the set. Quantitative measurement of the distribution of geometric properties over a set of features in a microstructure presents a significant challenge. Methods available for estimating size distributions are limited to very simple structures in which all of the features share the same quantitative shape.

\subsection{The topographic microstructural state}

Concepts in the quantitative microstructural state encompass properties of individual features and the global properties of feature sets. They provide no information about the spatial distribution of features in the microstructure; this class of information is specified in the topographical microstructural state. There are three primary categories of concepts in this level of description:

1. Gradients, which report systematic variations of geometric properties with position in the structure;

2. Anisotropies, which report systematic variations with orientation;

3. Associations, which report information about the position of features in the structure with respect to each other.

Gradients in microstructural properties typically arise when processing imposes a gradient in the influences like temperature, state of stress, atmosphere, and the like that operate to change the microstructure, Fig. 5a. Perhaps the most common example is provided in the surface treatment of materials; e.g., carburization or nitriding produce obvious microstructural gradients from the surface inward. If the global properties of the structure are of interest, then the presence of a gradient is a stereological sampling problem that must be properly averaged. Alternatively, the gradient may be quantified by taking measurements systematically at points along it. 
Anisotropies in the distribution of microstructural properties also arise from nonuniformities of the influences applied during processing, Fig. 5b. The most obvious example is the anisotropy of surface area in grain structures that arises during directional mechanical processing. Anisotropies also are common in solidification processing. As in the case of gradients, if the global properties of the structure are of interest, (e.g., the total surface area of grains in a deformed polycrystal) then the anisotropy presents itself as a sampling problem that must be appropriately averaged $^{14,15}$. On the other hand, the nature of the anisotropy may be characterized quantitatively by taking measurements in different directions ${ }^{16}$.

The concept of geometric associations may be applied to features from the same feature set, or alternatively, to features from different sets in the structure, Fig. 5c. A collection of particles of the $\beta$ phase may exhibit departure from a random spatial distribution by showing a tendency to cluster together (forming clumps in the limiting case) or to order (forming a regular lattice of particles like the $\gamma^{\prime}$ ordered phase common in many in nickel based superalloys). These tendencies may in principle be quantified by measuring the distribution of nearest neighbor distances. Such measurements have been applied on two dimensional sections ${ }^{17,18}$. Undertaking such measurements in three dimensions requires a reconstruction of the three dimensional structure from serial sections, confocal microscopy, acoustical microscopy or tomography ${ }^{17}$. Such approaches are not practical for opaque solid materials, where the only feasible approach, serial sectioning, requires an inordinate investment of effort ${ }^{4}$.

Features in one feature set may tend to associate with those of another in a microstructure, or to avoid them. An obvious example is shown in Fig. 5d where the oxide particles decorate the grain boundaries. Particles may tend to cluster on, or avoid, the grain edges in the structure. Measures of these tendencies are contained in contiguity measures ${ }^{19-21}$. These take the form of fractions of the value of the measure of extent of one feature set that is due to the association of another feature set. Consider for example a three phase structure, $\alpha \beta \gamma$. One measure of the tendency for the $\gamma$ phase to be associated with the $\alpha$ phase is the ratio of $\alpha \gamma$ surface area to the total boundary area of $\alpha$ particles, $(\alpha \beta+\alpha \gamma)$. The fraction of the length of grain edges in the $\alpha$ matrix occupied by $\beta$ particles is a measure of the tendency for $\beta$ particles to associate with grain edges. Other measures incorporate triple line lengths.

All of the concepts discussed in this section are fundamental in the sense that they have unambiguous meaning for features in arbitrary real three dimensional microstructures. All may be experimentally measured by applying the methods of stereology $y^{3,11,12}$. With a set of properly designed samples from probe populations each of the funda-

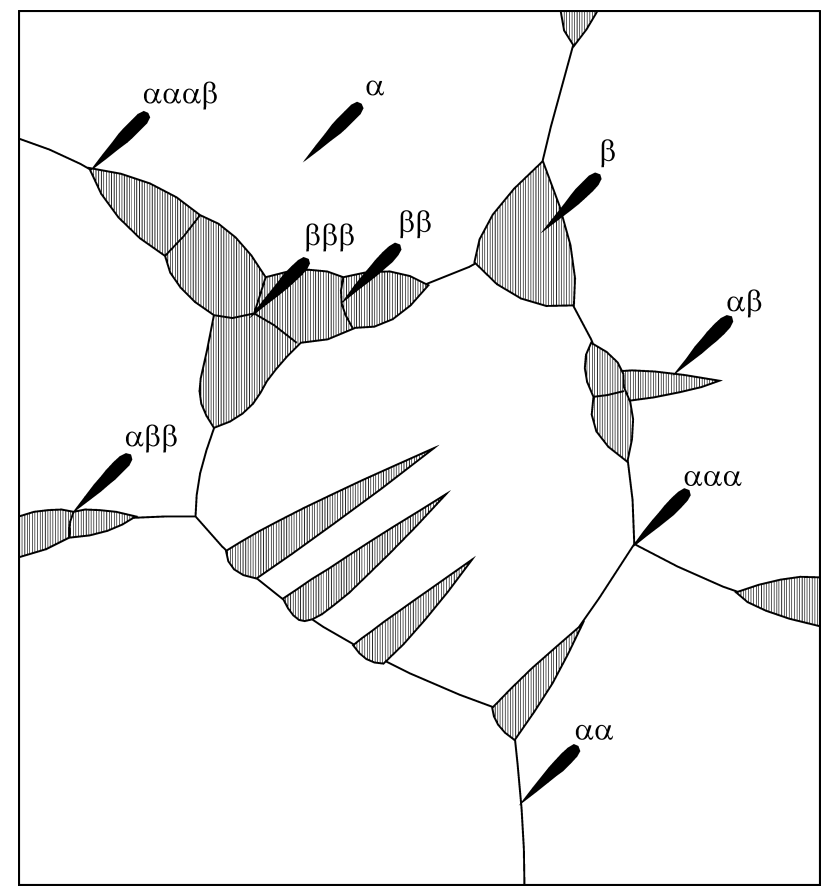

Figure 6. Two phase microstructure exhibiting all of the features in the two phase feature list: $\alpha, \beta, \alpha \alpha, \alpha \beta, \beta \beta, \alpha \alpha \alpha, \alpha \alpha \beta, \alpha \beta \beta, \beta \beta \beta$.

mental geometric properties listed in Table 1 may be estimated with statistical precision. For each geometric property, separate measurements may be made for all of the feature sets in the qualitative microstructural state that have that property. For example, for the structure shown in Fig. 6, all of the properties: $\mathrm{V}_{\mathrm{v}}{ }^{\alpha}, \mathrm{V}_{\mathrm{v}}{ }_{\mathrm{v}}, \mathrm{S}_{\mathrm{v}}{ }^{\alpha \alpha}, \mathrm{S}_{\mathrm{v}}{ }^{\alpha \beta}, \mathrm{S}_{\mathrm{v}}{ }^{\beta \beta}, \mathrm{L}_{\mathrm{v}}{ }^{\alpha \alpha \alpha}$, $\mathrm{L}_{\mathrm{v}}{ }^{\alpha \alpha \beta}, \mathrm{L}_{\mathrm{v}}{ }^{\alpha \beta \beta}$ and $\mathrm{L}_{\mathrm{v}}{ }^{\beta \beta \beta}$, may be stereologically estimated.

\section{Thermodynamics in Microstructural Design}

Classical phenomenological thermodynamics provides the basis for organizing information about the behavior of matter. The laws of thermodynamics lead to a general criterion for equilibrium expressed as an extremum principle: "the entropy of an isolated system is a maximum at equilibrium" "13. This extremum principle is the basis for a general strategy for finding conditions for equilibrium in the most complex kind of system. These conditions for equilibrium are sets of equations that must be obeyed in order for a system to be in its equilibrium state. Variables in these equations are specific properties of the system: temperatures, pressures, chemical potentials. Definitions for these properties, and the associated means for their measurement, arise in the development of the apparatus for describing the complex kinds of systems that are commonplace in materials science.

In its application to materials science the goal of thermodynamics is the calculation of equilibrium states. If one takes system I in state A and places it in environment $\mathrm{E}$ it 
will change toward a final, equilibrium condition predicted to be system II with state B. In phenomenological thermodynamics this goal has been realized for very general classes of systems: multicomponent, multiphase, open, reacting systems in which capillarity effects, crystal defects, elastic effects and external fields may play a role ${ }^{13}$. In the following sections discussion is limited to the two major application of thermodynamics in the engineering of microstructures:

1. Thermodynamics of phase diagrams; and

2. Thermodynamics of capillarity effects.

Phase diagrams provide the context within which processes occur and microstructures develop. Capillarity effects explain how the thermodynamics of the phase diagram operates through the geometry of the microstructure.

\subsection{Thermodynamics of phase diagrams}

The collection of equilibrium states for any system is represented as a map of the domains of stability of the various phase forms that a system may exhibit in a space that represents ranges of interest of the fundamental overarching variables, temperature, pressure and composition. Phase diagrams, once determined by direct observation of the phases displayed in a survey of combinations of compositions and temperatures for a system, are now derived from an integrated iteration of thermodynamic information and direct observation ${ }^{22}$. Further, first principle calculations of phase diagrams from phase stability and solution formation behavior derived from interactions modeled on the atomic scale ${ }^{23}$ are becoming increasingly successful.

As mentioned before, phase diagrams play a central role in the engineering of microstructures because they provide the context within which processes occur. In any attempt to understand what happened, or to predict what will happen, when microstructure I was subjected to treatment $\mathrm{E}$ and became microstructure II the first step should be to look at the phase diagram. However, phase diagrams present patterns of equilibrium states in temperature-pressurecomposition space. They provide information only about the ultimate state toward which a system tends. A phase diagram does not provide the path that the system will follow in its pursuit of equilibrium, although in simple cases the path may be strongly indicated. The rate at which the path is traversed by the system is also beyond the application of phase diagrams. The path, the competing kinetic processes that determine it, and its associated rate require another level of detail and sophistication in terms of the information required. Kinematic and kinetic considerations in microstructural design are treated in more detail in later sections.

The modern trend for constructing phase diagrams by combining direct structural observations with thermodynamic data and first principle modeling is resulting in a tool for engineering of microstructures with perhaps unforseen power. An early objective in these developments was to provide a means for using all of the data available for a given system in order to construct an optimal, reliable and consistent phase diagram for that system ${ }^{22}$. A second objective was the use of models to predict behavior in ternary and higher order systems by extending successful models for the binary systems involved. The investment required to produce experimental phase diagram information for quaternary and higher order systems makes such an endeavor impractical. Yet many materials of crucial technological importance are truly "multicomponent". It seems likely that systematic studies of such higher order systems must rely heavily upon theoretically calculated phase diagrams.

Perhaps the greatest potential that will be derived from this marriage of theory and experiment will be the application of the thermodynamic information underlying a successfully constructed phase diagram. The free energy / composition / temperature / pressure relationships that describe the energetics that determine the pattern of winners in the competition to determine domains of phase stability constitute information that is invaluable to an understanding of the kinetic processes that compete. In the first instance this information also determines the competition for the metastable phases that may exist, and provide quantitative information about how metastable these phases may be. The variation of free energy difference between competing phases with composition and temperature provides the pattern of "driving forces" that play a key role in most kinetic processes. This kind of quantitative energetic information is only now beginning to become available for systems beyond the binary. This is a case in which the information being developed has the potential to exceed our abilities to make use of it until we learn how to apply it in multicomponent systems.

The strategies and procedures developed for computing phase diagrams from interactions at the atomic level provides access to kinds of information not experimentally available. For example by incorporating elastic stresses and strains into the thermodynamic models it is possible to develop "coherent phase diagrams" 23 . In processes that involve ordering or spinodal decomposition the early stages produce particles that are coherent with the surrounding crystal lattice, i.e., the phase that forms and the parent phase share the same crystal lattice. In this case significant elastic energy is developed in accommodating the phases and the predicted domains of stability may be significantly different from those observed in bulk systems. These elastic effects must be incorporated into the evaluation of the driving forces for the kinetic processes that occur. 


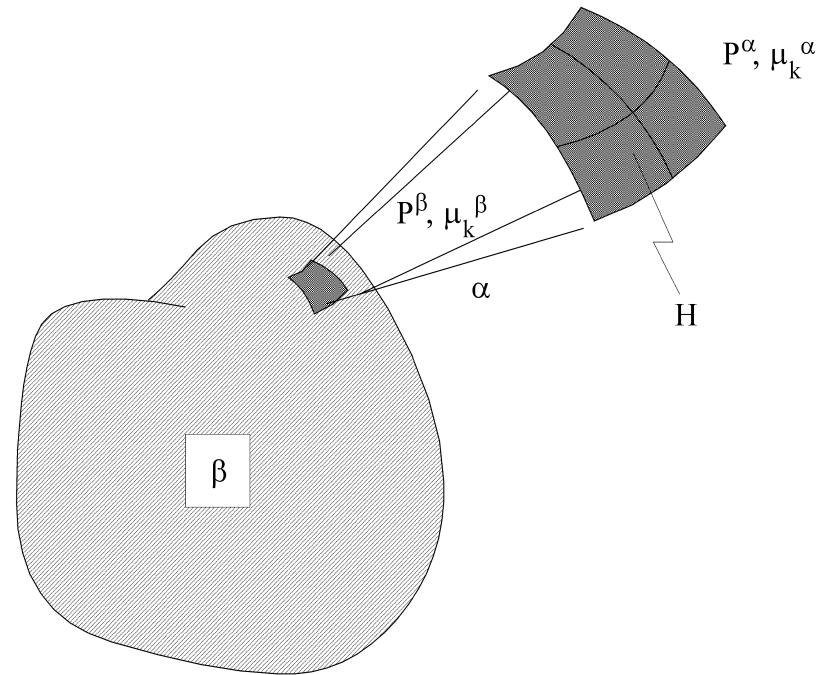

Figure 7. The condition for local equilibrium across a curved $\alpha \beta$ interface requires that the pressure in the $\beta$ phase be different from the pressure in the a phase by the factor $2 \gamma \mathrm{H}$ where $\gamma$ is the surface free energy and $\mathrm{H}$ is the local mean curvature of the surface. The chemical potencial of any component, $\mu_{\mathrm{k}}$, is the same on both sides of the interface.

\subsection{Thermodynamics of capillarity effects}

A microstructure is a spatial distribution of geometric entities: interfaces, triple lines, quadruple points. The subset of the thermodynamic apparatus that incorporates these geometric entities is called capillarity. The conditions for equilibrium involving geometric aspects of microstructure fall into two main scenarios:

1. Equilibrium at a curved interface where the pressure difference across the interface is proportional to the surface free energy and the local mean curvature, Fig. 7; and

2. Equilibrium at triple lines where a balance of the energies of the three meeting surfaces determines the local configuration, Fig. 8.

Both of these effects play important roles in determining microstructural evolution and thus in engineering microstructures.

The mechanical effect described in the first case can be shown to translate into shifts in phase boundaries on phase

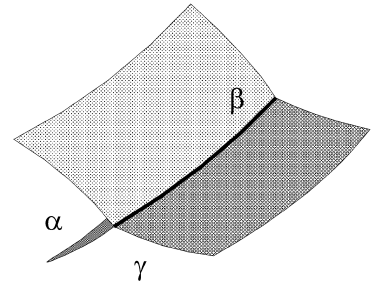

(a)

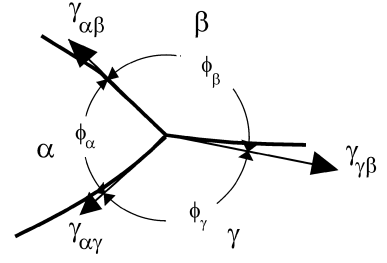

(b)
Figure 8. The condition for equilibrium at a triple line where three surfaces meet (a) determines the angle between the surfaces (b). These angles are measures of the tendencies for the phases to wet each other and may strongly influence the microstructure. diagrams. These translations then operate to influence processes involving those phases. The thermodynamic effects appear as an increase in vapor pressure for curved liquid/vapor interfaces, a decrease in the melting point for curved solid/liquid interfaces, shifts in vacancy concentrations for crystal/vapor interfaces, or shifts in composition for solid/solid interfaces involving two or more components. These "capillarity shifts" may act as the driving force for processes, as in coarsening, grain growth or sintering, may modify the driving force as in diffusion controlled growth, or may act as a barrier to be overcome, as in nucleation. These microstructural processes cannot be described without invoking the thermodynamics of capillarity.

Equilibrium at triple lines tends to have an obvious effect on microstructural geometry. The dihedral angles that develop in balancing the surface energies of the meeting interfaces determine "wetting tendencies". These aspects of the thermodynamics of interfaces appear in soldering and joining (where wetting is desirable) and in liquid phase penetration (where it is to be avoided). They play a crucial role in nucleation processes, favoring heterogeneous nucleation at grain boundaries, triple lines or quadruple points, or on dispersed inoculants or container walls.

Capillarity thermodynamics and the energetics of phase diagrams are key components in the quest to engineer microstructures. We are only now beginning to understand the operation of these parts of the thermodynamic apparatus in multicomponent systems.

\section{Kinematics and the Path of Microstructural Change}

In mechanics kinematics means the description of the geometry of motion (as in the motion of a compound lever system) without regard to the physical influences that cause the motion. For example in kinematics it is shown that the curve traced out by a point on a wheel as the wheel rolls along a line is a cycloid. The time variation of the spatial distribution of the components in a mechanical system may be described in terms of velocities of specific components, e.g., the displacement of a pin in the mechanism, or the angular rotation velocity of a lever.

\subsection{The kinematic equations}

There also exists a kinematics of microstructural evolution. In this case, the motions are visualized as the distribution of velocities over a moving interphase interface, Fig. 9. These motions produce changes in the geometry of the phases involved. A set of kinematic equations relate changes in the geometric properties of the microstructure to this velocity distribution.

Where $\mathrm{V}, \mathrm{S}$ and $\mathrm{M}$ are the volume, surface area and integral mean curvature of the phase bounded by the mov- 


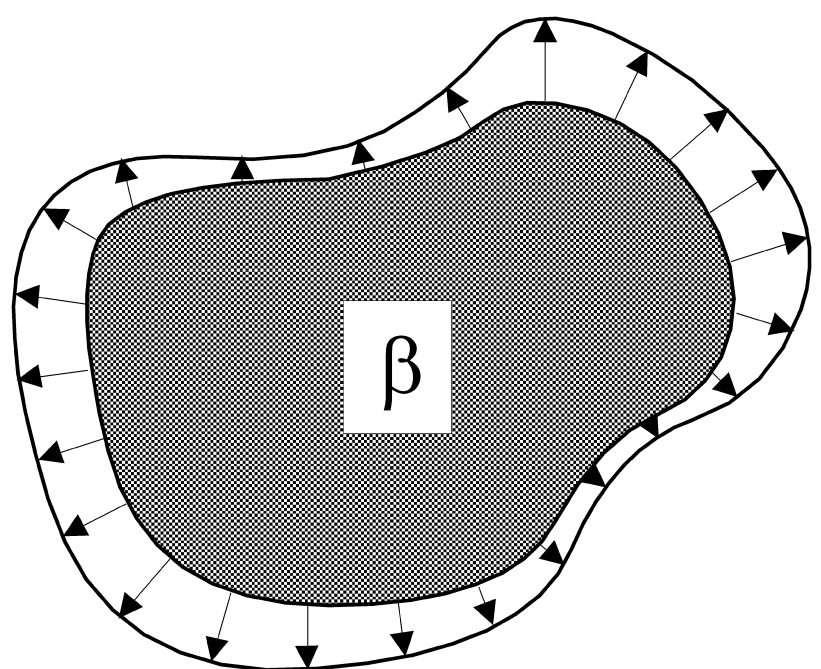

Figure 9. The distribution of velocities over the interface that bounds a feature illustrated in this figure is related to the changes in the geometric properties of the feature through the kinematic equations.

ing interface. $\mathrm{H}$ is the local mean curvature and $\mathrm{K}$ the Gaussian curvature of an element of boundary moving with velocity v. These equations make no simplifying assumptions about the geometry of the structure ${ }^{3}$.

$$
\begin{aligned}
& \frac{\mathrm{dV}}{\mathrm{dt}}=\iint_{S} \mathrm{v} \mathrm{dS} \\
& \frac{\mathrm{dS}}{\mathrm{dt}}=2 \iint_{S} \mathrm{v} \mathrm{H} \mathrm{dS} \\
& \frac{\mathrm{dM}}{\mathrm{dt}}=\iint_{\mathrm{S}} \mathrm{v} \mathrm{d} S
\end{aligned}
$$

The path of microstructural evolution is visualized as the sequence of geometric states that a system exhibits during a microstructural process. The path may be made quantitative by measuring the geometric properties of the microstructure at a sequence of observations that sample the process. An individual quantitative microstructural state may be visualized as a point in a space that has a number of dimensions equal to the number of geometric parameters that have been quantified. The path is then a space curve in that multidimensional space, connecting subsequent states along the process. The path provides rudimentary information about the process that is occurring that is not available from observations of the time variation of individual geometric properties.

If the boundary motions are smooth and continuous some information about what determines the path may be obtained from ratios of the kinematic equations:

$$
\begin{aligned}
& \frac{d S}{d V}=\frac{2 \iint_{S} v H d S}{\iint_{S} v d S} \\
& \frac{d M}{d V}=\frac{\iint_{S} v K d S}{\iint_{S} v d S}
\end{aligned}
$$

The sequence of geometric states, expressed in terms of how the surface area and integral mean curvature vary with the volume of the phase, is seen to be determined in a complex way by ratios of integrals over the area of the moving interface of weighted measures of the velocity distribution on the moving interface.

\subsection{Topological processes in microstructural evolution}

Most microstructural paths result from two classes of local processes:

1. The smooth migration of interfacial elements under the control of local atomic accommodations. These produce changes in the metric properties of the structure and are governed by the kinematic equations.

2. Discontinuous changes in the structure that alter the topology of the microstructure. The time frame for these changes is short compared with those associated with smooth boundary migration.

Smooth boundary migrations produce changes that are global because they act at every point on the moving interface. Topological processes are by their nature local, affecting only elements of the microstructure in their immediate area. Both classes of processes contribute significantly to determining the path of microstructural change.

The coupled operation of topological and metric processes is a common phenomenon in microstructural evolution. Typically the structure changes smoothly as interfaces migrate; this migration sets up a local shape change that reaches a critical condition and, on a short time scale, the topological process occurs. Some familiar examples follow.

Grain growth results from the disappearance of grains in a space filling polycrystal, a topological process which decreases the number of grains in the microstructure. (A second topological process in which grains switch neighbors is responsible for maintaining the distribution of grain topologies in the system.) The geometric configuration necessary for these topological events to occur is set up by the smooth and continuous migration of grain boundaries until locally some critical condition has been attained and a topological change occurs.

Sintering is characterized by topological changes that disconnect the pore network until pores become isolated; if 
the microstructural context is correct, these isolated pores then disappear. These topological events are set up by the smooth migration of pore-solid interface until some local critical geometric condition is attained.

Coarsening involves smooth boundary migration, with interfaces bounding large particles moving outward and those bounding small particles moving inward. The latter ultimately results in a topological process, the disappearance of small particles. As a result, the number of particles decreases and the average particle size increases.

Precipitation begins with a topological process, the formation of new particles in nucleation. Further microstructural development derives from growth, which is the smooth and continuous migration of the surface bounding precipitate particles.

The topological processes are by their nature instantaneous. Topological events are disruptions in the otherwise smooth evolution of the geometry of the structure. A cluster of atoms attains a critical size and a new particle is defined to be nucleated. A small grain shrinks inward on itself until it disappears in grain growth or coarsening. A channel in a pore network reaches a critical configuration and at some instant in time pinches closed, further disconnecting the pore network. Rates of change of local values of the metric properties may be large in the vicinity of a topological event immediately before or after it occurs. However, global metric properties change smoothly and continuously as they average over contributions from these events.

Qualitatively, the path is characteristic for a given class of process (e.g., grain growth, coarsening, etc.) The quantitative path is then determined by the choices of processing variables applied to the system. For example, in precipitation increasing the supersaturation may increase the nucleation rate relative to the growth rate and alter the path to produce a structure with a larger number of particles that are on the average smaller in size. The precipitation process thus exhibits an envelope of paths. This envelope of paths displays the ensemble of microstructural states that are accessible to the process. The choice of the processing variables selects a specific path from this envelope of paths and narrows the focus to microstructural states along that path. Interruption of the process at the appropriate time leaves the structure in a selected microstructural state.

\section{The Kinetics of Microstructural Evolution}

The kinematic equations describe the global geometric changes of a microstructure in terms of the distribution of interface velocities. The kinematic equations become kinetic equations when the interface velocity function $\mathrm{v}$ is evaluated from physical considerations including the thermodynamic context and the atomic processes that make the interface move. In general the local velocity of an element of moving interface is governed physically by an interface accommodation equation ${ }^{24,25}$. A phase boundary exists because the two

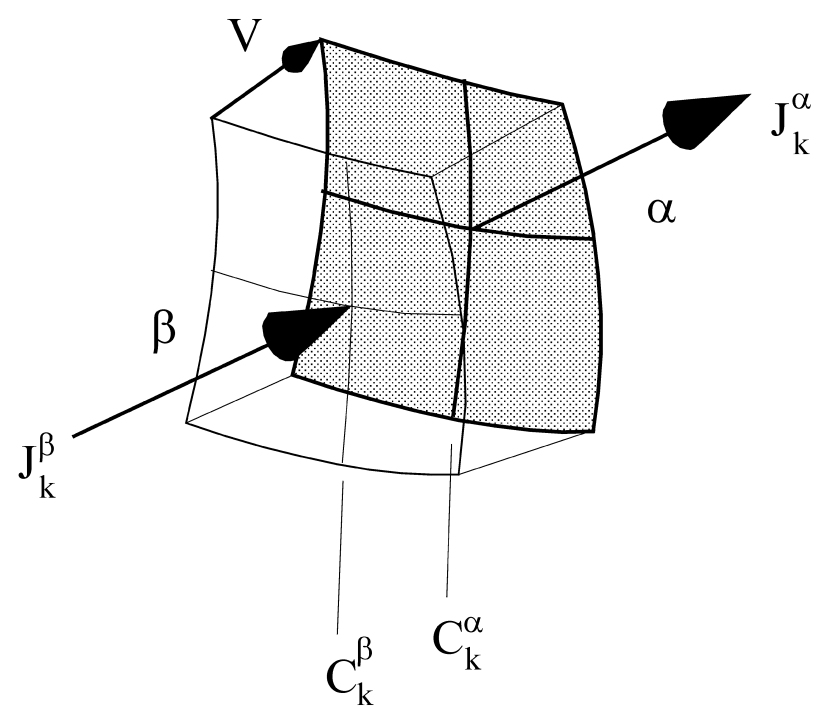

Figure 10. Illustration of the factors involved in the interface accommodation equation that describes the velocity $\mathrm{v}$ of a phase of the boundary between two phases.

phases that define it have different properties. In order for the boundary to move, these differences must be accommodated. This is generally achieved through local flows of chemical components (to accommodate composition differences) or heat (to accommodate enthalpy differences).

To illustrate the kinetic evaluation of the local velocity $\mathrm{v}$, consider the case of a boundary between two binary phases, $\alpha$ and $\beta$, that have two different compositions at the interface, $\mathrm{C}^{\alpha}$ and $\mathrm{C}^{\beta}$, Fig. 10. In order for the interface to move this concentration difference must be accommodated by supplying (or removing) atoms of the components. In this illustration let it be assumed that the motion of the interface is controlled by the supply of these atoms by diffusion in the adjacent phases. If the process is controlled by this diffusion then local equilibrium obtains at the interface and $C^{\alpha}$ and $C^{\beta}$ are molar concentrations of the component (say component B) computed from the equilibrium tie line for $\alpha$ and $\beta$ at the temperature of the interface in the phase diagram for the system. Define $\mathrm{J}^{\alpha}$ and $\mathrm{J}^{\beta}$ to be the diffusion fluxes of component $\mathrm{B}$ (moles of $\mathrm{B} / \mathrm{cm}^{2}-\mathrm{sec}$ ) at the interface in the $\alpha$ and $\beta$ phases respectively. Then it can be shown ${ }^{24,25}$ that conservation of component $\mathrm{B}$ requires that

$$
v=\frac{J^{\beta}-J^{\alpha}}{C^{\beta}-C^{\alpha}}
$$

This is the compositional interface accommodation equation.

\subsection{Precipitation processes}

An alloy with composition $\mathrm{C}^{\mathrm{o}}$ is first solution treated at a high temperature where the phase diagram, Fig. 11, shows a single phase to be the equilibrium structure. This alloy is 


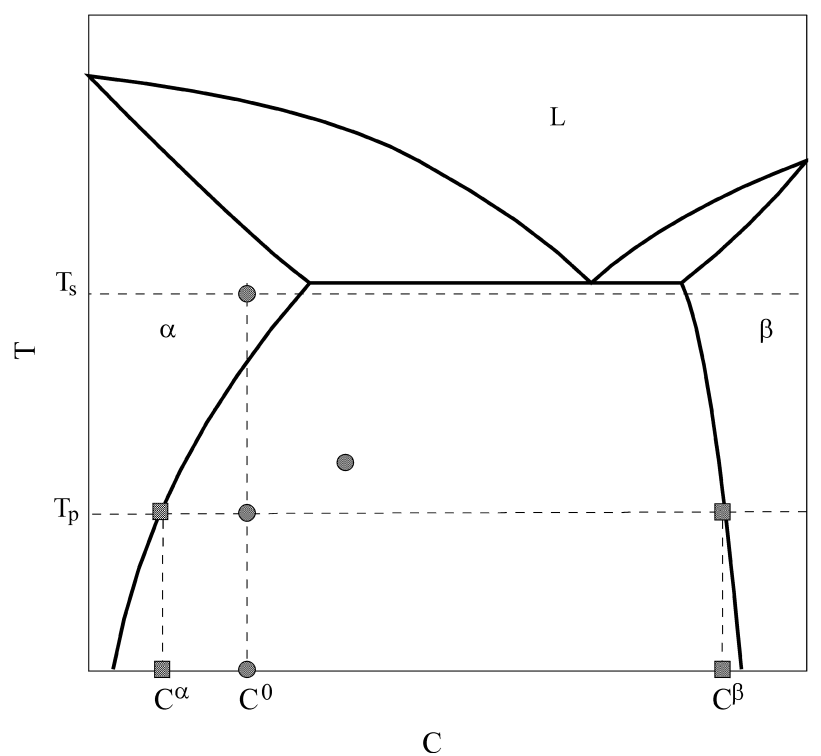

Figure 11. Typical phase diagram in which a precipitaion process can be made to occur.

then quenched to an aging temperature that lies within a two phase $\alpha+\beta$ field in the phase diagram. Particles of the $\beta$ phase nucleate and begin to grow. Typically the $\beta$ particles form and grow at a composition that is near their equilibrium composition, $\mathrm{C}^{\beta}$. With this condition, there is no gradient in the $\beta$ phase during growth: $\mathrm{J}^{\beta}=0$, so that

$$
v=-\frac{J^{\alpha}}{C^{\beta}-C^{\alpha}}
$$

The flux at the interface in the $\alpha$ phase can be expressed in terms of the concentration gradient at the interface, $\nabla \mathrm{C}$, and the diffusion coefficient in the $\alpha$ phase:

$$
\mathrm{J}^{\alpha}=-\mathrm{D} \Delta \mathrm{C}
$$

If the process is controlled by diffusion in the matrix phase then a concentration field will evolve with time in the matrix. This composition distribution is a solution to Fick's second law which governs diffusion determined by the initial conditions and the boundary conditions at the $\alpha \beta$ interface. This concentration field will exhibit flux lines that connect to the particle surfaces. A trace of the composition distribution along such a flux line is shown in Fig. 12. The gradient at the interface may be expressed in terms of the composition difference $\left(\mathrm{C}^{\mathrm{o}}-\mathrm{C}^{\alpha}\right)$ and a local diffusion length scale, $\lambda$, shown in Fig. 12.

$$
\Delta \mathrm{C}=\frac{\mathrm{C}^{0}-\mathrm{C}^{\alpha}}{\lambda}
$$

The diffusion length scale for any element of interface grows parabolically with the time since the particle nucleated, until concentration interference occurs in the diffu-

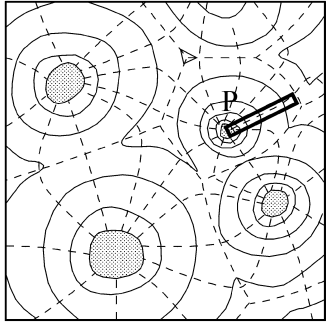

(a)

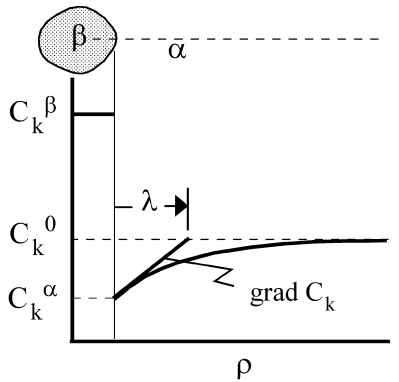

(b)
Figure 12. (a) Two dimensional sketch of a diffusion generated concentration field for $\beta$ precipitating in $\alpha$, showing the isoconcentration contours and flux lines. (b) For any point $\mathrm{P}$ on the $\alpha \beta$ interface the concentration gradient in the $\alpha$ along the flux line can be described by a composition difference $\left(\mathrm{C}_{\mathrm{k}}{ }^{0}-\mathrm{C}_{\mathrm{k}}{ }^{\alpha}\right)$ and a diffusion lenght scale, $\lambda$.

sion fields between neighboring particles. Thereafter the growth rate of the length scale slows down. The growth rate approaches zero as the length scales approach infinity and the system approaches equilibrium. The distribution of diffusion length scales at any time in this process is tied to the time dependence of the nucleation rate since the length scale for each surface element starts from zero at its moment of nucleation.

The interface accommodation equation for diffusion controlled growth can thus be written

$$
\mathrm{v}=\frac{\mathrm{D}}{\mathrm{C}^{\beta}-\mathrm{C}^{\alpha}} \frac{\mathrm{C}^{0}-\mathrm{C}^{\alpha}}{\lambda}=\mathrm{D}\left[\frac{\mathrm{C}^{0}-\mathrm{C}^{\alpha}}{\mathrm{C}^{\beta}-\mathrm{C}^{\alpha}}\right] \frac{1}{\lambda}
$$

The factor in brackets, which is the ratio of the composition difference in the matrix phase to the composition difference across the phase boundary, is called the supersaturation ratio for the process, and can be determined from the tie line on the phase diagram that describes this two phase system. $\mathrm{D}$ is the diffusivity in the matrix phase at the composition $\mathrm{C}^{\alpha}$, and $\lambda$ is the local diffusion length scale in the matrix at a surface element.

In a microstructure that is evolving by diffusion controlled growth of a $\beta$ phase into an $\alpha$ matrix the interface velocity, $\mathrm{v}$, is expected to vary with position over the $\alpha \beta$ interface. Inspection of Eq. (9) reveals that all of that variation is embodied in the diffusion length scale, $\lambda$. Thus, the distribution of diffusion length scales in the structure plays a central role in determining the path and kinetics of the evolution of the microstructure.

Rates of change of the global geometric properties can be related to the local physics embodied in Eq. (9) by inserting this expression for the interface velocity into the kinematic equations. The rate of change of the volume of the growing $\beta$ phase is given by 
change of the global geometric properties can be related to the local physics embodied in Eq. (9) by inserting this expression for the interface velocity into the kinematic equations. The rate of change of the volume of the growing $\beta$ phase is given by

$$
\begin{gathered}
\frac{\mathrm{dV}}{\mathrm{dt}}=\iint_{\mathrm{S}} \mathrm{vdS}=\iint_{\mathrm{S}} \mathrm{D}\left[\frac{\mathrm{C}^{0}-\mathrm{C}^{\alpha}}{\mathrm{C}^{\beta}-\mathrm{C}^{\alpha}}\right] \frac{1}{\lambda} \mathrm{dS}= \\
\mathrm{D}\left[\frac{\mathrm{C}^{0}-\mathrm{C}^{\alpha}}{\mathrm{C}^{\beta}-\mathrm{C}^{\alpha}}\right] \iint_{\mathrm{S}} \frac{1}{\lambda} \mathrm{dS} \\
\frac{\mathrm{dV}}{\mathrm{dt}}=\mathrm{D}\left[\frac{\mathrm{C}^{0}-\mathrm{C}^{\alpha}}{\mathrm{C}^{\beta}-\mathrm{C}^{\alpha}}\right]<\frac{1}{\lambda}>\mathrm{S}^{\mathrm{S}}
\end{gathered}
$$

where $<\frac{1}{\lambda}>\mathrm{S}$ is a surface area weighted harmonic mean of the distribution of diffusion length scales. The kinetics of growth of the $\beta$ phase is clearly determined by the temperature of the process through the diffusion coefficient in the $\alpha$ phase, which is strongly temperature sensitive, and the supersaturation ratio obtained from the phase diagram, which may not be as sensitive to temperature. The nucleation part of the precipitation process is implicit in the distribution of diffusion length scales. If nucleation is in the site saturation mode (all of the available nucleation sites are activated at $t=0$ ) then the distribution of diffusion length scales will be very narrow, at least until the late stages of the process where concentration interference becomes dominant. As nucleation becomes time dependent, this distribution broadens.

The time dependence of surface area and integral mean curvature can be determined by inserting the velocity Eq. (9) into their kinematic Eqs. (3):

$$
\frac{\mathrm{d} S}{\mathrm{dt}}=2 \mathrm{D}\left[\frac{\mathrm{C}^{0}-\mathrm{C}^{\alpha}}{\mathrm{C}^{\beta}-\mathrm{C}^{\alpha}}\right]<\frac{1}{\lambda}>\mathrm{M}^{\mathrm{M}}
$$

and, for the change in $\mathrm{M}$

$$
\frac{\mathrm{dM}}{\mathrm{dt}}=\mathrm{D}\left[\frac{\mathrm{C}^{0}-\mathrm{C}^{\alpha}}{\mathrm{C}^{\beta}-\mathrm{C}^{\alpha}}\right]<\frac{1}{\lambda}>\Omega^{\Omega}
$$

Insight into the path of microstructural change for a microstructure evolving by diffusion controlled growth of the $\beta$ phase can be obtained by taking ratios of these kinetic equations. The projection of the path which plots the surface area of the microstructure versus the volume of the growing phase is given by the ratio

$$
\frac{d S}{d V}=\frac{2 D\left[\frac{C^{0}-C^{\alpha}}{C^{\beta}-C^{\alpha}}\right]<\frac{1}{\lambda}>M^{M}}{D\left[\frac{C^{0}-C^{\alpha}}{C^{\beta}-C^{\alpha}}\right]<\frac{1}{\lambda}>S^{S}}=2 \frac{<\frac{1}{\lambda}>M^{M}}{<\frac{1}{\lambda}>S^{S}}
$$

The component of the path represented by the variation of the integral mean curvature with the volume of the growing particles is

$$
\frac{\mathrm{dM}}{\mathrm{dV}}=\frac{\mathrm{D}\left[\frac{\mathrm{C}^{0}-\mathrm{C}^{\alpha}}{\mathrm{C}^{\beta}-\mathrm{C}^{\alpha}}\right]<\frac{1}{\lambda}>\Omega^{\Omega}}{\mathrm{D}\left[\frac{\mathrm{C}^{0}-\mathrm{C}^{\alpha}}{\mathrm{C}^{\beta}-\mathrm{C}^{\alpha}}\right]<\frac{1}{\lambda}>\mathrm{S}^{\mathrm{S}}}=\frac{<\frac{1}{\lambda}>\Omega^{\Omega}}{<\frac{1}{\lambda}>\mathrm{S}^{\mathrm{S}}}
$$

In both of these equations the dependence upon the diffusion coefficient in the matrix, D, and the phase diagram concentrations contained in the supersaturation ratio cancel. The path depends upon the ratio of differently weighted averages of the diffusion length scale distribution, and current values of geometric properties, S, M and $\Omega$. The breadth of the diffusion length scale distribution is determined by the time dependence of the nucleation behavior in the process, i.e., by the rate of topological change. It is concluded that the variability of the path of microstructural evolution in diffusion controlled precipitation is largely determined by the kinetics of its topological nucleation process: nucleation

All of these results reflect the microstructology viewpoint: the description is carried as far as possible before introducing simplifying geometric assumptions.

In order to apply the kinematic equations to specific microstructural processes it is necessary to visualize the distribution of concentration gradients in the matrix at the moving interface. This evaluation is specific to each class of process.

\subsection{Diffusion controlled coarsening}

Coarsening sets in when the chemical driving force for growth of a precipitate phase has been exhausted so that the much smaller differences in composition associated with capillarity effects may begin to operate. In this case the thermodynamics of capillarity effects predicts that the concentration in the $\alpha$ phase at a surface element is determined by the local mean curvature of the element ${ }^{13}$. An element of surface on a small particle has a relatively high concentration in the adjacent $\alpha$ phase; surface elements on coarse particles have a relatively small concentration in the adjacent $\alpha$ phase. Thus, there exists a distribution of concentrations over the collection of boundaries of the $\alpha$ phase. If the process is controlled by diffusion between $\beta$ particles through the matrix $\alpha$ phase, then a concentration field evolves with time in this process. This evolution is governed by Fick's second law and the distribution of concentrations at the $\alpha \beta$ surfaces that bound the $\alpha$ phase.

The composition field will exhibit flux lines (normal to the isocomposition contours) that connect any given surface element with a communicating neighbor element. Fig. 13 sketches a composition profile along such a flux line. 

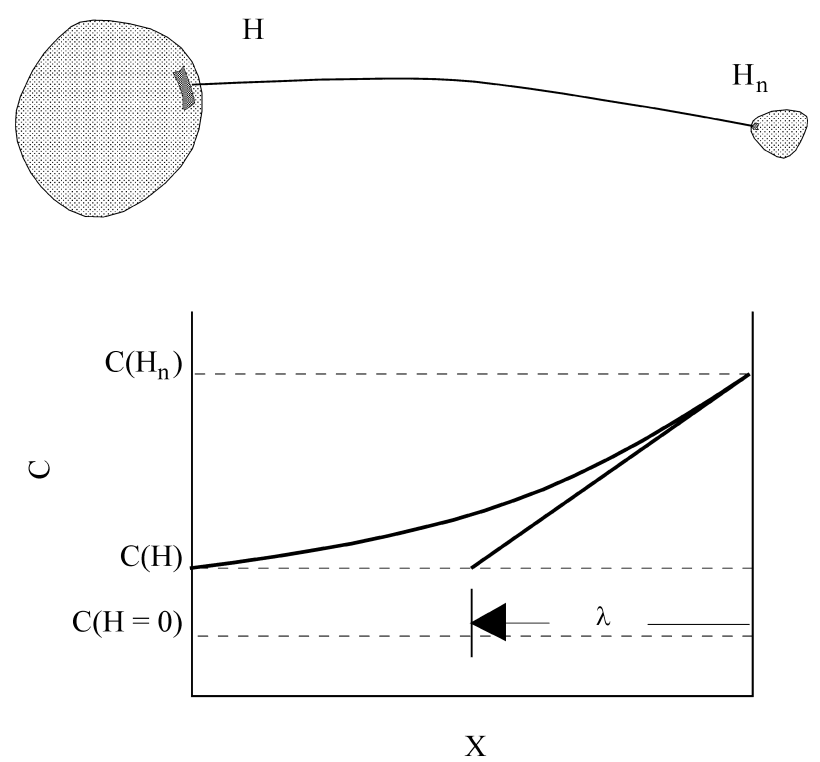

Figure 13. Illustration of the concentration profile along a flux line that joins two "communicating neighbors" surface elements and the associated diffusion lenght scale $\lambda$ in diffusion controlled coarseing.

The gradient at the interface for the smaller particle can be visualized in terms of the composition difference between the surface elements (which is determined by the difference in their mean curvatures) and the diffusion length scale, $\lambda$ :

$$
\Delta \mathrm{C}=\frac{\mathrm{C}(\mathrm{H})-\mathrm{C}(\mathrm{H})_{\mathrm{n}}}{\lambda}=\frac{2 \gamma \mathrm{V}^{\beta}}{\mathrm{R} \mathrm{T}} \frac{\mathrm{H}_{\mathrm{n}}-\mathrm{H}}{\lambda}
$$

In this result $\gamma$ is the surface free energy of the interface, $\mathrm{V}^{\beta}$ is the molar volume of the $\beta$ phase, $\mathrm{R}$ is the gas constant, $\mathrm{T}$ the absolute temperature $\mathrm{H}_{\mathrm{n}}$ the mean curvature of the communicating neighbor element and $\mathrm{H}$ that of the reference element. Insert this evaluation into the interface accommodation equation:

$$
\begin{aligned}
& \mathrm{v}=\frac{\mathrm{D}}{\mathrm{C}^{\beta}-\mathrm{C}^{\alpha}} \Delta \mathrm{C}=\frac{\mathrm{D}}{\mathrm{C}^{\beta}-\mathrm{C}^{\alpha}} \frac{2 \gamma \mathrm{V}^{\beta}}{\mathrm{R} \mathrm{T}} \frac{\mathrm{H}_{\mathrm{n}}-\mathrm{H}}{\lambda} \\
& \mathrm{v}=\mathrm{K}_{\mathrm{D}} \frac{\mathrm{H}_{\mathrm{n}}-\mathrm{H}}{\lambda}
\end{aligned}
$$

Note that if $\mathrm{H}$ is larger than $\mathrm{H}_{\mathrm{n}}$ (implying the reference element is on a smaller particle) $\mathrm{v}$ is negative and the particle shrinks. For the opposite case, the particle grows. Thus, in diffusion controlled coarsening the local velocity of an element is determined by its curvature, $\mathrm{H}$, that of its communicating neighbor, $\mathrm{H}_{\mathrm{n}}$, and a diffusion length scale, $\lambda$ which is of the order of the distance between particles.

Insertion of this result into the kinematic equations gives the predictions for the time evolution of global geometric properties for diffusion controlled coarsening. For this process the total volume of the $\beta$ phase does not change so that $\mathrm{dV} / \mathrm{dt}=0$. It can be shown that this condition yields the result that the average curvature of the communicating neighbor elements is simply the average mean surface curvature in the system $<\mathrm{H}>$ defined in Eq. (2). Insertion of this result into the remaining kinematic equations yields predictions of the rate of change of surface area and total curvature for diffusion controlled coarsening. In this case the integrations involved lead to weighted averages of the mean surface curvature distribution, as well as the distribution of diffusion length scales. These results contain no simplifying geometric assumptions. For a complete presentation of these results see reference ${ }^{26}$.

The path of microstructural change for coarsening processes is presumed to be highly constrained. Classical theories on the subject suggest that, after some adjustment to the initial particle size distribution, all coarsening systems (of any given volume fraction) approach a fixed size distribution, and thus a fixed path of microstructural evolution. Factors that might alter the path, such as the spatial distribution of the particles (whether clustered or ordered) have been left unexplored.

\subsection{Grain growth}

The local process that governs grain growth is grain boundary migration. In contrast with the previously described processes, grain boundary migration does not involve concentration fields, Fick's second law or diffusion length scales. The driving force for grain growth is the decrease in surface energy associated with the elimination of grain boundary area. This is accomplished if each element of boundary moves toward its local center of curvature. The classic kinetic model, due to von Neuman ${ }^{27}$ and Mullens $^{28}$, describes grain growth in a two dimensional grain structure. They assumed that the velocity of the boundary element is proportional to its local curvature:

where $\alpha$ is a rate constant that depends upon temperature and composition and $\mathrm{k}$ is the local curvature of the boundary. This assumption leads to the classical " $6-\mathrm{n}$ " rule: that rate of change of the area of any grain depends only upon the number of sides that is has and the rate constant $\alpha$. No other geometric factors are involved ${ }^{4}$. The corresponding hypothesis for grain growth in three dimensional microstructures is that the velocity is proportional to the local mean curvature $\mathrm{H}$ of the grain boundary.

$$
\mathrm{v}=-\alpha \mathrm{k}
$$

The von Neumann result and its three dimensional analog neglect the topological processes that are an integral part of grain growth ${ }^{30,31}$. The mean grain volume, which is determined by the number of grains in the structure and no other geometric parameter, can only change as a result of the topological event in which a small grain disappears, Fig. 14. As small grains are removed from the system new small 


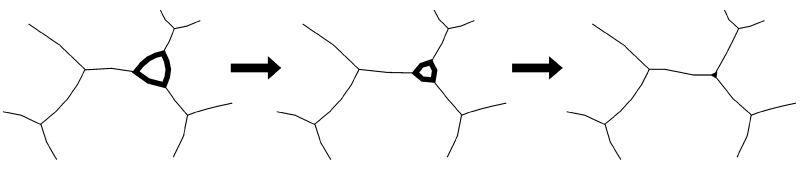

Type I: Grain Annihilation

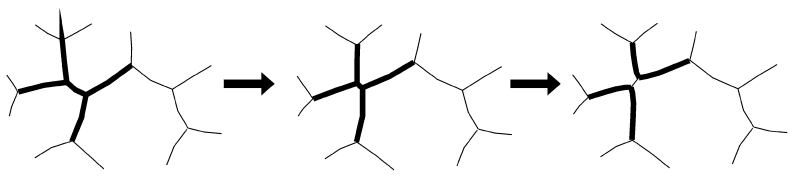

Type II: Switching Process

Figure 14. Topological processes that occur in two dimensional grain growth: (a) the disappearance of small grains, and (b) the switching process.

grains are formed as a result of the topological switching processes illustrated in Fig. 14. In the vicinity of the catastrophic topological events the surface curvatures are large and the interface velocities correspondingly high. Thus, in grain growth most interface elements are migrating slowly toward their centers of curvature but, at any instant in time, a small fraction of the surface elements that happen to be near a topological event, are moving very rapidly. The kinematic equations may be used in principle to predict kinetics and the path of microstructural change. However, it is necessary to incorporate the observation that much of the change in surface area of the grain boundary network that occurs in any time interval is more or less directly associated with the topological events that occur in that time interval. Indeed, in an analysis of grain growth in two dimensions $^{29}$ it has been estimated that over $90 \%$ of the change in boundary area is a direct result of the topological processes.

Paths of microstructural change in grain growth range from "normal grain growth" in which the structure coarsens without changing the relative width of the grain size distribution, to "abnormal grain growth" or "secondary recrystallization" where selected grains grow at a rate far exceeding their neighbors. It seems evident that this range of paths is governed by the spatial distribution of the topological events. In normal grain growth the topological events are uniformly distributed in space. In abnormal grain growth these processes apparently tend to be clustered near abnormal grains. Contributing factors include textural effects, nonuniformity in the distribution of second phase particles or nonuniformity in the developing "normal" grain size distribution. The material and process variables that control the path of microstructural change in grain growth remain a subject for experimental study.

\subsection{Sintering}
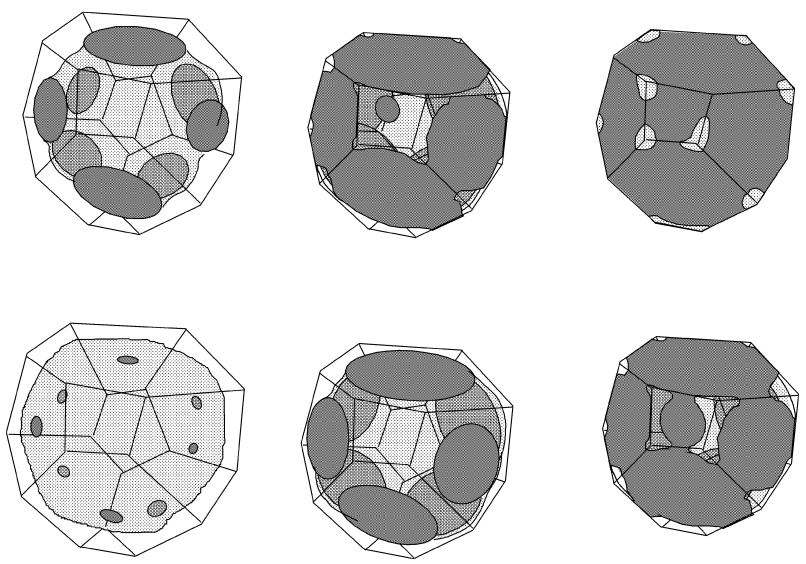

Figure 15. Illustraiting the progress in terms of the evolution of the pore and grain boundary structures associated with a single grain.

A geometrically general approach to modeling the sintering process requires modification of the kinematic equations. In sintering the migration of the pore solid interface is not the only contribution to the changes in the geometric properties. The annihilation of vacancies at grain boundaries plays a significant role in densification which also contributes to changes in other geometric properties. Thus integrals of the local velocity over the moving pore solid interface do not adequately describe the geometric changes that occur.

When a stack of particles is heated to a significant fraction of their melting point, necks form at points of contact. These necks are grain boundaries in the solid phase. The pore phase is a connected network which interpenetrates the solid connected network. The necks grow until they impinge, forming triple lines in the developing grain boundary network. The formation of triple lines is accompanied by the pinching off of channels in the pore network. Eventually a fully connected grain boundary network results as the pore network disconnects into isolated pores.

The thermodynamics of defect structures combines with capillarity theory to predict that vacancies are generated at the curved pore surface. These vacancies migrate to nearby grain boundaries between particles. As the vacancies annihilate at the grain boundaries, the grain centers move toward each other and the porous aggregate densifies.

The process may be visualized in terms of a space filling cell structure in which each cell contains one grain and its associated porosity ${ }^{32}$. Each cell face is partly covered by grain boundary, partly by porosity. Let $f$ be the ratio of cell face area to grain boundary area at any point in the process. When the equivalent of one layer of vacancies is annihilated in the grain boundary on a particular face the volume of the cell is decreased by the removal of a layer one atom thick from the whole cell face. In the early stages of sintering, when the grain boundary occupies a small frac- 
tion of a cell face, annihilation of a layer of vacancies in the grain boundary carries with it a large volume change, i.e., the vacancy annihilation process is efficient. In the late stages of the process after porosity is isolated $\mathrm{f}$ approaches 1 and the volume change of the structure is roughly equal to the volume of vacancies annihilated.

Sintering is an unusual microstructural process in that it involves two networks with evolutions that are coupled ${ }^{32}$. Assume for simplicity that the particles are single crystals. At the outset the pore network is completely connected and the grain boundary network, which is confined to the interparticle necks, is completely disconnected. As densification proceeds and pore channels begin to pinch off as growing necks impinge on each other, disconnected portions of the grain boundary structure begin to connect to form subnetworks. At a still later stage the grain boundary subnetworks connect up to form a complete network. This grain boundary network is capable of the metric and topological changes that characterize grain growth. In this stage of sintering, which probably characterizes the range from 15 to $5 \%$ porosity, the grain boundary network may become uncoupled from the pore network. The subsequent path of microstructural change is strongly dependent upon the balance between grain growth and pore evolution because the elimination of porosity is most efficient for pores in the grain boundary network. The attainment of a fully dense body is favored if the pore structure and the grain boundary network remain coupled. If the grain structure coarsens, either normally or abnormally, pores left without contact to the grain boundary network become stable and resist elimination.

Paths of microstructural evolution in sintering are circumscribed primarily by the geometry of the initial powder stack embodied in the particle size, size distribution, shapes and stacking ${ }^{4}$. Processing scenarios that involve precompaction or hot pressing versus loose stack sintering significantly alter the path in the neck growth and channel closure stages of the process but have less effect in the late stages of the process where the pores are isolated. In that stage the coupling between the grain growth process and pore evolution determines the path.

\section{Summary}

Engineering of microstructures requires wise choices based upon knowledge on the one hand of the relation between processing and the microstructural states that may be developed and, on the other hand, of the relation between microstructural states that may be achieved and their properties. This presentation has focused on the tools that are needed to choose a material and design a process to achieve a target microstructural state.

Concepts of microstructural state and the path of microstructural change form the basis for the engineering of microstructures. For a given material and initial micros- tructural state each class of process yields an envelope of microstructural paths. Fundamental geometric concepts must be mastered in order to describe microstructural states and paths qualitatively, then quantitatively.

A knowledge of the thermodynamics that underlie multicomponent, multiphase systems leads to an understanding of phase diagrams for real, complex systems. These maps of the domains of stability of possible equilibrium states for a system form the context within which microstructural processes occur.

A set of kinematic equations relate the evolution of microstructural geometry to the distribution of velocities on boundaries in the structure. These geometrically general relationships provide a basis for establishing paths of microstructural change and rates of traverse of those paths.

These kinematic equations may be converted to kinetic equations by applying the principles of both reversible and irreversible thermodynamics to endow local interface velocities with a physical description. This connection with the physics of the moving atoms is the basis for establishing the effect that processing sequence and variables have upon the path and kinetics of microstructural evolution.

Many common microstructural processes have a topological component. The typical topological event is a local change in connectivity or number of some geometric entity in the structure. It may be accompanied by relatively large changes in local geometric properties. Rates of topological processes relative to rates of metric property changes may be responsible for introducing breadth into the spread of possible microstructural paths that a system may choose.

Engineering of microstructure presumes that a target microstructure has been circumscribed which exhibits a combination of properties desired for a particular application. The target microstructural state must be produced from a selected material through processing. It is a particular state obtained by interrupting a microstructural evolution that carries the material along some microstructural path. The spectrum of microstructural paths available from a given starting state is determined by the processing sequence and processing variables. Understanding these connections so that the right material may be selected and subjected to the right process requires mastery of the geometry, thermodynamics, kinematics and kinetics of microstructural evolution.

\section{References}

1. Rhines, F.N. Microstructology, Behavior of Microstructure of Materials. Dr. Riederer-Verlag, GmbH, Stuttgart, Germany, 1886.

2. DeHoff, R.T. Topography of microstructure. Metallography, n. 8, p. 71-87, 1975.

3. DeHoff, R.T.; Rhines, F.N. Quantitative Microscopy. McGraw-Hill, New York, NY, p. 291-325, 1968. 
4. Aigeltinger, E.H.; DeHoff, R.T. Quantitative determination of topological and metric properties during sintering of copper. Met. Trans., n. 6, p.1853-1857, 1975.

5. DeHoff, R.T.; Rhines, F.N. Quantitative Microscopy. McGraw-Hill, New York, NY, p. 307-316, 1968.

6. Minkowski, H. Math.Ann., n. 57, p. 447-452, 1903.

7. DeHoff, R.T. The quantitative estimation of mean surface curvature. Trans. Met. Soc. AIME, n. 239, p. 617-621, 1967.

8. DeHoff, R.T. Geometrical meaning of the integral mean curvature. Microstructural Science Volume 5, Elsevier-North Holland, New York, p. 331-343, 1977.

9. DeHoff, R.T. Integral mean curvature and platelet growth. Met. Trans., n. 10A, p. 1948-1949, 1979.

10. DeHoff, R.T.; Gehl, S.M. Quantitative microscopy of lineal features in three dimensions. Proc. $4^{\text {th }}$ Intl. Cong. For Stereology, National Bureau of Standards, Gaithersburg, MD, p. 29-40, 1975.

11. Underwood, E.E. Quantitative Stereology, AddisonWesley Pub. Co., Reading, MA, p. 41, 1970.

12. Kurzydlowski, K.J.; Ralph, B. The Quantitative Description of the Microstructure of Materials, CRC Press, Boca Raton, FL p. 275, 1995.

13. DeHoff, R.T. Thermodynamics in Materials Science. McGraw-Hill, New York, NY, p. 355-383, 1993.

14. Hilliard, J.E. Specification and measurement of microstructural anisotropy. Trans. Met. Soc. AIME, n. 224, p. 1201-1207, 1962.

15. Baddeley, A.J.; Gundersen, H.J.G.; Cruz-Orive, L.M. Estimation of surface from vertical sections. J. of Microscopy, n. 142, p. 259-271, 1986.

16. Underwood, E.E. Quantitative Stereology, AddisonWesley Pub. Co., Reading, MA, p. 49-79, 1970.

17. Russ, J.C. Computer Assisted Microscopy. Plenum Press, New York, NY, p. 261-264, 1990.

18. Schwarz, H.; Exner, H.E. The characterization of the arrangement of feature centroids in planes and volumes. J. of Microscopy, n. 129, p. 155-169, 1983.

19. Gurland, J. The measurement of grain contiguity in two phase alloys.Trans. Met. Soc. AIME, n. 212, p. 452-457, 1958.
20. Cahn, J.W.; Hilliard, J.E. The measurement of grain contiguity in opaque samples. Trans. Met. Soc. AIME, n. 224, p. 759-765, 1959.

21. Underwood, E.E. Quantitative Stereology, AddisonWesley Pub. Co., Reading, MA, p. 99-103, 1970.

22. Kattner, U.R. The thermodynamic modeling of multicomponent phase equilibria. Journal of Metals, p. 14 - 19, December, 1977.

23. Fontaine, D. de. From Gibbsian thermodynamics to electronic structure: nonempirical studies of alloy phase equilibria, MRS Bulletin, p. 16 - 25, August, 1996.

24. Sekerka, R.F.; Jeanfils, C.L.; Heckel, R.W. Lectures on the Theory of Phase Transformations, Aaronson, H.I., ed., AIME, New York, NY p. 117-132, 1975.

25. Geiger, G.H.; Pourier, D.R. Transport Phenomena in Metallurgy, Addison-Wesley, Reading, MA, p. 491496, 1973.

26. DeHoff, R.T. A geometrically general theory for diffusion controlled coarsening. Acta Metallurgica, n. 39, p. 2349-2360, 1991.

27. Neumann, J. von. Curvature driven grain growth in two dimensions. Metal Interfaces, ASM Seminar, ASM, Materials Park, OH, p.108-118, 1952.

28. Mullins, W.W. Role of grain boundary grooving in grain growth in two dimensions. J. Appl. Phys., n. 27, p. 900-905, 1956.

29. DeHoff, R.T. Metric and topological contributions to the rate of change of boundary length in two dimensional grain growth. Acta.Mat., in press.

30. Smith, C.S. Grain shapes and other metallurgical applications of topology. Metal Interfaces, American Society for Metals, Cleveland, OH, p. 95-108, 1952.

31. Craig, K.R.; Rhines, F.N. Mechanisms of steady state grain growth in aluminum. Met. Trans., n. 5, p. 413419, 1974.

32. DeHoff, R.T. A Stereological model of sintering. Science of Sintering. New Directions for Materials Processing and Microstructural Control, Uskokovic, D.P.; Palmour III, H.; Spriggs, R.M., eds., Plenum Press, New York, p. 55-72, 1989. 